\title{
The validity of the Euler-Lagrange equation for solutions to variational functionals with fast growth
}

\section{Agnese Caielli and Arrigo Cellina}

\begin{abstract}
For $L$ convex and defined on $\mathbb{R}^{N}$, we consider a solution $u$ to the problem of minimizing $\int_{\Omega} L(\nabla v(x)) d x$. We provide a growth condition on $L$ to guarantee that $u$ is locally bounded and, by building suitable variations, we prove the validity of the Euler-Lagrange equation without imposing differentiability on $L$.
\end{abstract}

Keywords: Calculus of variations, Euler-Lagrange equation, boundedness of the solution.

MSC2010: $49 \mathrm{~K} 10$

\section{Introduction}

The main necessary condition satisfied by a solution $u$ to a variational problem is the Euler-Lagrange equation: this equation is the starting point to establish further regularity properties of the solution, as the higher differentiability of the solution itself. However, in spite of the importance of this equation, its validity for the solution to a general variational problem is yet to be established, in particular for functionals having fast growth, and progress in this area is slow. Recently, Degiovanni and Marzocchi [2], with a clever construction of the variations to be used in the problem, succeeded in proving the validity of the Euler-Lagrange equation for variational problems of the kind

$$
\operatorname{minimize} \int_{\Omega} L(\nabla v(x)) d x \text { on } u+W_{0}^{1,1}(\Omega)
$$

where $\Omega$ is an open subset of $\mathbb{R}^{N}$ and $\xi \rightarrow L(\xi)$ is a convex and differentiable function, under the assumption of the local boundedness of solution $u$.

Considering the problem of the validity of the Euler-Lagrange equation from a different point of view, in [1], the authors introduced a method to avoid the assumption of differentiability of the convex function $L$ to establish the validity of the EulerLagrange equation. This method is based on the application of the Hahn-Banach 
and of the Riesz Representation theorems, and, so far, required the Lagrangian $L$, appearing in the functional to be minimized, to grow at most exponentially.

The purpose of the present paper is to bring together the different techniques of [2] and of [1] and to prove the validity of the Euler-Lagrange equation (in the form that will be discussed below) for problem (1.1), assuming the fast growth of $L$ but without assuming differentiability.

\section{Notations, growth assumptions and the statement of the main result}

We consider $\mathbb{R}^{N}$ with the Euclidean norm $|\cdot|$ and unit ball $\mathbb{B}$. The ball about 0 and radius $R$ is $R \mathbb{B}$. The unit vector of the $j$-th coordinate axis is $e_{j}$. Given a closed convex $K \subset \mathbb{R}^{N}$, by $m_{K}$ we mean the unique point of $K$ of minimal norm and by $\|K\|$ we mean $\sup \{|k|: k \in K\} . I_{A}(\cdot)$ is the indicator function of the set $A$ and $\omega_{N}$ is the volume of the unit ball. $f^{*}$ is the polar or Fenchel transform [3] of $f$. Without loss of generality, we shall assume that $L$ takes values in $\mathbb{R}^{+} . \partial L$ is the subdifferential of the convex function $L$. Under the assumptions of the present paper, $\partial_{\xi} L(\xi)$ is a non-empty compact convex subset of $\mathbb{R}^{N}$ and the map $\xi \mapsto \partial_{\xi} L(\xi)$ is an upper semicontinuous set valued map. Given a solution $u$, the shorthand notation $D_{L}(x)$ means the set $\partial_{\xi} L(\nabla u(x))$.

A solution $u$ to problem (1.1) is a function $u$ in $W^{1,1}(\Omega)$ such that $\int_{\Omega} L(\nabla u(x)) d x$ $<+\infty$ and such that

$$
\int_{\Omega} L(\nabla u(x)) d x \leq \int_{\Omega} L(\nabla v(x)) d x
$$

for $v$ in $u+W^{1,1}(\Omega)$, where the integral at the right hand side can assume the value $+\infty$.

In what follows it is essential that conditions be provided in order to make sure that the solution $u$ is in $\mathrm{L}_{l o c}^{\infty}(\Omega)$. The following form of the Sobolev imbedding Theorem is classical: assume that, for some positive $k$, we have

$$
L(\xi) \geq k \frac{1}{p}|\xi|^{p}
$$

with $p>N$. Then, $L(\nabla u) \in \mathrm{L}_{l o c}^{1}(\Omega)$ implies that $u$ is continuous, hence that $u \in \mathrm{L}_{l o c}^{\infty}(\Omega)$.

Since, for the purpose of the present paper, only the local boundedness of $u$ is required, we shall instead assume the following condition. 
Growth condition (GC). There exist a function $\mathbb{L}: \mathbb{R} \rightarrow \mathbb{R}^{+}, \mathbb{L}(0)=0$, such that $L(\xi) \geq \mathbb{L}(|\xi|)$ and

$$
\int_{0^{+}} \mathbb{L}^{*}\left(\frac{1}{t^{N-1}}\right) \cdot t^{N-1} d t<\infty .
$$

Proposition 2.1. Let L satisfy (2.1), then it satisfies (GC). Moreover, there exists $L$ satisfying $(G C)$ but not (2.1).

Proof. We have

$$
\mathbb{L}^{*}(|z|)=\left(k \frac{1}{p}|\cdot|^{p}\right)^{*}(|z|)=k\left(\frac{1}{p}|\cdot|^{p}\right)^{*}\left(\frac{|z|}{k}\right)=k \frac{1}{q}\left(\frac{|z|}{k}\right)^{q}
$$

with $\frac{1}{p}+\frac{1}{q}=1$, so that $(N-1)(q-1)<1$ and

$$
\frac{1}{k^{q-1}} \frac{1}{q} \int_{0}^{\delta}\left(\frac{1}{t^{N-1}}\right)^{q} t^{N-1} d t=\frac{1}{k^{q-1}} \frac{1}{q} \int_{0}^{\delta} \frac{1}{t^{(N-1)(q-1)}} d t<\infty
$$

so that (GC) holds. Conversely, let $r>0, N=2$ and define

$$
\mathbb{L}^{*}(|z|)=\frac{|z|^{2}}{|\ln | z||^{1+r}}
$$

so that, for $0<\delta<1$,

$$
\int_{0}^{\delta} \mathbb{L}^{*}\left(\frac{1}{t}\right) t d t=\int_{0}^{\delta} \frac{1}{t(|\ln t|)^{1+r}} d t=\left.\frac{1}{r} \frac{1}{(|\ln t|)^{r}}\right|_{0} ^{\delta}<\infty
$$

and set $L(z)=\mathbb{L}(|z|)=\mathbb{L}^{* *}(|z|)$. We claim that for no $k>0$ and $p>2$ we can have $L(\xi) \geq k \frac{1}{p}|\xi|^{p}$ for all $\xi$. Assume that such $k$ and $p$ exist. We have

$$
\frac{|z|^{2}}{|\ln | z||^{1+r}}=\mathbb{L}^{*}(|z|) \leq\left(k \frac{1}{p}|\cdot|^{p}\right)^{*}(|z|)=k\left(\frac{1}{p}|\cdot|^{p}\right)^{*}\left(\frac{|z|}{k}\right)=k \frac{1}{q}\left(\frac{|z|}{k}\right)^{q}
$$

with $q<2$, a contradiction.

The following is the local boundedness result.

Theorem 2.2. Let $L$ be convex and satisfy Condition (GC). Let $u \in W^{1,1}(\Omega)$ be such that $\int_{\Omega} L(\nabla u(x)) d x<+\infty$. Then, $u \in \mathrm{L}_{l o c}^{\infty}(\Omega)$.

Proof. Set $M_{1}=\int_{\Omega} L(\nabla u(x)) d x$. Fix an open $\omega \subset \subset \Omega$ and let $h$ be such that, for every $x \in \omega, B(x, 2 h) \subset \Omega$. Set $M_{2}=\int_{0}^{h} \mathbb{L}^{*}\left(\frac{1}{t^{N-1}}\right) t^{N-1} d t$. Let $\left(\rho_{n}\right)$ be a sequence 
of standard mollifiers with support in $B\left(0, \frac{1}{n}\right)$, with $\frac{1}{n}<h$, and, on $\omega+h \mathbb{B}$, set $u_{n}=\rho_{n} * u$. Then, $u_{n} \rightarrow u$ in $\mathrm{L}^{1}(\omega+h \mathbb{B})$ and

$$
\int_{\omega+h \mathbb{B}} L\left(\nabla u_{n}(x)\right) d x=\int_{\omega+h \mathbb{B}} L\left(\int_{B\left(0, \frac{1}{n}\right)} \rho_{n}(y) \nabla u(x-y) d y\right) d x ;
$$

by Jensen's inequality,

$$
\begin{gathered}
\int_{\omega+h \mathbb{B}} L\left(\nabla u_{n}(x)\right) d x \leq \int_{\omega+h \mathbb{B}} \int_{B\left(0, \frac{1}{n}\right)} \rho_{n}(y) L(\nabla u(x-y)) d y d x \\
=\int_{B\left(0, \frac{1}{n}\right)} \rho_{n}(y) \int_{\omega+h \mathbb{B}} L(\nabla u(x-y)) d x d y \leq \int_{B\left(0, \frac{1}{n}\right)} \rho_{n}(y) \int_{\Omega} L(\nabla u(x)) d x d y=M_{1} .
\end{gathered}
$$

In particular, we obtain that $\int_{\omega+h \mathbb{B}} \mathbb{L}\left(\left|\nabla u_{n}(x)\right|\right) d x \leq M_{1}$.

Fix arbitrarily $x \in \omega$ and consider polar coordinates with the origin in $x$. We have

$$
u_{n}(x)=u_{n}(0, \theta)=u_{n}(r, \theta)-\int_{0}^{r} \frac{d}{d t} u_{n}(t, \theta) d t
$$

hence, for $0<r<h$,

$$
\left|u_{n}(x)\right| \leq\left|u_{n}(r, \theta)\right|+\int_{0}^{h}\left|\nabla u_{n}(t, \theta)\right| d t
$$

so that

$$
\begin{gathered}
\int_{\partial B(0,1)} \int_{0}^{h} r^{N-1}\left|u_{n}(x)\right| d r d H^{N-1} \leq \int_{\partial B(0,1)} \int_{0}^{h} r^{N-1}\left|u_{n}(r, \theta)\right| d r d H^{N-1} \\
\quad+\int_{\partial B(0,1)} \int_{0}^{h} r^{N-1} \int_{0}^{h}\left|\nabla u_{n}(t, \theta)\right| d t d r d H^{N-1} .
\end{gathered}
$$

We obtain

$$
\left|u_{n}(x)\right| h^{N} \omega_{N} \leq \int_{B(x, h)}\left|u_{n}(y)\right| d y+\frac{h^{N}}{N} \int_{\partial B(0,1)} \int_{0}^{h}\left|\nabla u_{n}(r, \theta)\right| d r d H^{N-1}
$$

Multiply and divide the final integrand by $r^{N-1}=|x-y|^{N-1}$ to obtain

$$
\frac{h^{N}}{N} \int_{\partial B(0,1)} \int_{0}^{h}\left|\nabla u_{n}(r, \theta)\right| d r d H^{N-1}=\frac{h^{N}}{N} \int_{B(x, h)} \frac{\left|\nabla u_{n}(y)\right|}{|x-y|^{N-1}} d y .
$$

By polarity,

$$
\int_{B(x, h)} \frac{\left|\nabla u_{n}(y)\right|}{|x-y|^{N-1}} d y \leq \int_{B(x, h)} \mathbb{L}\left(\left|\nabla u_{n}(y)\right|\right) d y+\int_{B(x, h)} \mathbb{L}^{*}\left(\frac{1}{|x-y|^{N-1}}\right) d y
$$




$$
\leq M_{1}+\int_{\partial B(0,1)} \int_{0}^{h} \mathbb{L}^{*}\left(\frac{1}{t^{N-1}}\right) t^{N-1} d t d H^{N-1} \leq M_{1}+M_{2} N \omega_{N} .
$$

In addition, since $u_{n} \rightarrow u$ in $\mathrm{L}^{1}(\omega+h \mathbb{B})$, it is bounded in $\mathrm{L}^{1}(\omega+h \mathbb{B})$ : there exists $M_{3}$ such that $\int_{B(x, h)}\left|u_{n}(y)\right| d y \leq M_{3}$, any $x \in \omega+h \mathbb{B}$. Then, (2.3) yields

$$
\left|u_{n}(x)\right| \leq \frac{1}{h^{N} \omega_{N}}\left(M_{3}+\frac{h^{N}}{N}\left[M_{1}+M_{2} N \omega_{N}\right]\right)=K
$$

and the right hand side is independedent on $x$, hence the estimate holds on $\omega$. A subsequence of the $\left(u_{n}\right)$ converges to $u$ pointwise almost everywhere, so that almost everywhere on $\omega$ we have

$$
|u(x)| \leq K
$$

In the case where $L$ is not necessarily differentiable, the suitable form of the Euler-Lagrange equations satisfied by a solution $u$ to problem 1.1 should be: There exists $p(\cdot) \in\left(\mathrm{L}_{l o c}^{1}(\Omega)\right)^{N}$, a selection from $\partial L(\nabla u(\cdot))$, such that $\operatorname{div} p(\cdot)=0$ in the sense of distributions. The main purpose of the present paper is to prove the following theorem. In its statement, a condition on the size of $\partial L$ appears; when $L$ is differentiable, this condition is always satisfied taking $h_{0}=0$ and $h_{1}=1$

Theorem 2.3. Let $\Omega$ be an open subset of $\mathbb{R}^{N}$; let $L: \mathbb{R}^{N} \rightarrow \mathbb{R}^{+}$be convex, and let the growth condition (GC) be satisfied. In addition, assume that there exist two nonnegative constants $h_{0}$ and $h_{1}$ such that, for every $\xi$, we have $\sup _{k \in \partial L(\xi)}<k, \xi>\leq$ $h_{0}+h_{1}\left|\inf _{k \in \partial L(\xi)}<k, \xi>\right|$. Let $u$ be a solution to problem (1.1). Then there exists $p \in \mathrm{L}_{\text {loc }}^{1}(\Omega)$, a selection from the map $x \rightarrow \partial L(\nabla u(x))$, such that, for every $\eta \in C_{c}^{\infty}(\Omega)$, we have

$$
\int_{\Omega}\langle p(x), \nabla \eta(x)\rangle d x=0
$$

\section{The construction of variations and preliminary results}

The main difficulty in proving the validity of the Euler-Lagrange equation for integral functionals with fast growth lies in the fact that, given a solution $u$ and a variation $\eta \in C_{c}^{\infty}(\Omega)$, in general the map $x \rightarrow L(\nabla u(x)+\nabla \eta(x))$ is not in $\mathrm{L}_{l o c}^{1}(\Omega)$. This fact requires a more careful construction.

Let $\eta \in C_{c}^{\infty}(\Omega)$ and let $K$ be a compact subset of $\Omega$ with $\operatorname{supt}(\eta) \subseteq \operatorname{int}(K)$. Since $u \in \mathrm{L}_{l o c}^{\infty}(\Omega)$, there exists $R>0$ such that $|u|<R$ on $K$. Following [2], consider 
the following families of variations: for every $t>0$, set:

$$
\bar{v}_{t}(x)=\left\{\begin{array}{lll}
\max \{\operatorname{t\eta }(x)-R, u(x)\} & \text { if } \quad x \in K \\
u(x) & \text { if } \quad x \in \Omega \backslash K
\end{array}\right.
$$

and

$$
\underline{v}_{t}(x)= \begin{cases}\min \{\operatorname{t\eta }(x)+R, u(x)\} & \text { if } \quad x \in K \\ u(x) & \text { if } \quad x \in \Omega \backslash K \\ & \end{cases}
$$

Let $A$ be an open bounded subset of $\Omega$ such that $K \subset A$ : we recall that $W^{1, p}(A)$, with $1 \leq p \leq \infty$, is a lattice-ordered Banach space, and we have $\bar{v}_{t}, \underline{v}_{t} \in u+W_{0}^{1,1}(\omega) \cap$ $\mathrm{L}_{l o c}^{\infty}(\omega)$ for every $\omega \subset \subset A$, and

$$
\begin{aligned}
& \nabla \bar{v}_{t}(x)= \begin{cases}\nabla u(x) & \text { if } u \geq t \eta-R \vee x \in \Omega \backslash K \\
t \nabla \eta(x) & \text { otherwise }\end{cases} \\
& \nabla \underline{v}_{t}(x)= \begin{cases}\nabla u(x) & \text { if } u \leq t \eta+R \vee x \in \Omega \backslash K \\
t \nabla \eta(x) & \text { otherwise. }\end{cases}
\end{aligned}
$$

From the above remarks, it follows that $L\left(\nabla \bar{v}_{t}\right)$ and $L\left(\nabla \underline{v}_{t}\right)$ belong to $L^{1}(\Omega)$.

Let $K$ be a compact subset of $\Omega$ and let $K^{\prime}, K^{\prime \prime}$ be two additional compact subsets of $\Omega$ with $K \subseteq \operatorname{int}\left(K^{\prime}\right) \subseteq K^{\prime} \subseteq \operatorname{int}\left(K^{\prime \prime}\right)$. Choose $R$ so that $|u|<R$ on $K^{\prime \prime}$. Define the following variation $v$ : let $\vartheta \in C_{c}^{\infty}(\Omega)$ be such that $0 \leq \vartheta \leq 1$ on $\Omega, \vartheta=1$ on $K$ and $\vartheta=0$ on $\Omega \backslash \operatorname{int}\left(K^{\prime}\right)$ and set

$$
v(x)= \begin{cases}\max \{R(2 \vartheta(x)-1), u(x)\} & \text { if } \quad x \in K^{\prime} \\ u(x) & \text { if } \quad x \in \Omega \backslash K^{\prime} .\end{cases}
$$

Then, as noted previously, $L(\nabla v) \in \mathrm{L}^{1}(\Omega)$ and $v \in u+W_{0}^{1,1}(\Omega) \cap \mathrm{L}_{l o c}^{\infty}(\Omega)$.

Finally, consider the following variations. Let $K, K^{\prime}, K^{\prime \prime}$ and $\vartheta$ as before.

Let $R>0$ be so large that, for any $1 \leq j \leq N$, we have $R(2 \vartheta(x)-1)+x_{j}>u$ on $K$ and $R(2 \vartheta(x)-1)+x_{j}<u$ on $K^{\prime \prime} \backslash \operatorname{int}\left(K^{\prime}\right)$. For $1 \leq j \leq N$, set

$$
v_{j}^{+}(x)=\left\{\begin{array}{lll}
\max \left\{R(2 \vartheta(x)-1)+x_{j}, u(x)\right\} & \text { if } \quad x \in K^{\prime} \\
u(x) & \text { if } \quad x \in \Omega \backslash K^{\prime}
\end{array}\right.
$$


Let $R>0$ be so large that, for any $1 \leq j \leq N$, we have $R(2 \vartheta(x)-1)-x_{j}>u$ on $K$ and $R(2 \vartheta(x)-1)-x_{j}<u$ on $K^{\prime \prime} \backslash \operatorname{int}\left(K^{\prime}\right)$ and set

$$
v_{j}^{-}(x)= \begin{cases}\max \left\{R(2 \vartheta(x)-1)-x_{j}, u(x)\right\} & \text { if } x \in K^{\prime} \\ u(x) & \text { if } x \in \Omega \backslash K^{\prime}\end{cases}
$$

Again, $v_{j}^{+}, v_{j}^{-} \in u+W_{0}^{1,1}(\Omega) \cap \mathrm{L}_{l o c}^{\infty}(\Omega)$ and both $L\left(\nabla v_{j}^{+}\right)$and $L\left(\nabla v_{j}^{-}\right)$are in $\mathrm{L}^{1}(\Omega)$. In particular, we have that, on $K^{\prime}, \nabla v_{j}^{+}=e_{j}$ and $\nabla v_{j}^{-}=-e_{j}$.

Recall that $D_{L}(x)$ is the set $\partial_{\xi} L(\nabla u(x))$. In what follows we shall need the following main Lemma.

Lemma 3.1. Let $u \in W_{l o c}^{1,1}(\Omega)$ be a solution and $v \in W_{l o c}^{1,1}(\Omega)$ be such that $L(\nabla v) \in$ $\mathrm{L}^{1}(\Omega)$. Then:

1. $\sup _{k \in D_{L}(x)}<k, \nabla(v-u)>\in \mathrm{L}^{1}(\Omega)$

2. $\int_{\Omega} \sup _{k \in D_{L}(x)}<k, \nabla(v-u)>d x \geq 0$.

Proof. Since $L$ is convex, it follows that for every $t \in[0,1]$

$$
L(\nabla u+t(\nabla v-\nabla u)) \leq L(\nabla u)+t[L(\nabla v)-L(\nabla u)]
$$

so that $L(\nabla u+t(\nabla v-\nabla u)) \in \mathrm{L}^{1}(\Omega)$. Rewrite inequality (3.8) as

$$
\frac{1}{t}[L(\nabla u+t(\nabla v-\nabla u))-L(\nabla u)] \leq L(\nabla v)-L(\nabla u)
$$

and, in particular,

$$
\left(\frac{1}{t}[L(\nabla u+t(\nabla v-\nabla u))-L(\nabla u)]\right)^{+} \leq(L(\nabla v)-L(\nabla u))^{+} .
$$

Since $L(\nabla v)-L(\nabla u) \in \mathrm{L}^{1}(\Omega)$, then $(L(\nabla v)-L(\nabla u))^{+} \in \mathrm{L}^{1}(\Omega)$ and

$$
\left(\frac{1}{t}[L(\nabla u+t(\nabla v-\nabla u))-L(\nabla u)]\right)^{+} \in \mathrm{L}^{1}(\Omega)
$$

Since (see [3]) $\left(\frac{1}{t}[L(\nabla u+t(\nabla v-\nabla u))-L(\nabla u)]\right)^{+}$converges pointwise decreasing to $\left.\sup _{k \in D_{L}(x)}<k, \nabla(v-u)>\right)^{+}$, applying the dominated convergence theorem, we obtain

$$
\int_{\Omega}\left(\sup _{k \in D_{L}(x)}<k, \nabla(v-u)>\right)^{+}=\lim _{t \rightarrow 0^{+}} \int_{\Omega}\left(\frac{1}{t}[L(\nabla u+t(\nabla v-\nabla u))-L(\nabla u)]\right)^{+} .
$$


On the other hand, by Fatou's lemma we also have

$$
\int_{\Omega}\left(\sup _{k \in D_{L}(x)}<k, \nabla(v-u)>\right)^{-} \leq \underline{\lim }_{t \rightarrow 0^{+}} \int_{\Omega}\left(\frac{1}{t}[L(\nabla u+t(\nabla v-\nabla u))-L(\nabla u)]\right)^{-}
$$

thus

$$
\int_{\Omega}-\left(\sup _{k \in D_{L}(x)}<k, \nabla(v-u)>\right)^{-} \geq \varlimsup_{t \rightarrow 0^{+}} \int_{\Omega}-\left(\frac{1}{t}[L(\nabla u+t(\nabla v-\nabla u)-L(\nabla u)])^{-}\right.
$$

and, combining (3.11) and the above inequality, we obtain

$$
\begin{gathered}
\int_{\Omega \in D_{L}(x)} \sup _{k, k} \nabla(v-u)>\geq \lim _{t \rightarrow 0^{+}} \int_{\Omega}\left(\frac{1}{t}[L(\nabla u+t(\nabla v-\nabla u))-L(\nabla u)]\right)^{+} \\
+\varlimsup_{t \rightarrow 0^{+}} \int_{\Omega}-\left(\frac{1}{t}[L(\nabla u+t(\nabla v-\nabla u))-L(\nabla u)]\right)^{-} \\
=\varlimsup_{t \rightarrow 0^{+}} \int_{\Omega} \frac{1}{t}[L(\nabla u+t(\nabla v-\nabla u))-L(\nabla u)] .
\end{gathered}
$$

Since $u$ is a solution, $\int_{\Omega} L(\nabla u) d x \leq \int_{\Omega} L(t \nabla v+(1-t) \nabla u) d x$ for every $t \in[0,1]$, thus proving assertion 2 of the Lemma.

We have shown that

$$
\int_{\Omega}\left(\sup _{k \in D_{L}(x)}<k, \nabla(v-u)>\right)^{-} \leq \int_{\Omega}\left(\sup _{k \in D_{L}(x)}<k, \nabla(v-u)>\right)^{+} .
$$

As we have noticed,

$$
\lim _{t \rightarrow 0^{+}} \frac{1}{t}[L(\nabla u+t(\nabla v-\nabla u))-L(\nabla u)]=\sup _{k \in D_{L}(x)}<k, \nabla(v-u)>
$$

and, from (3.9), we obtain

$$
\sup _{k \in D_{L}(x)}<k, \nabla(v-u)>\leq L(\nabla v)-L(\nabla u) \in \mathrm{L}^{1}(\Omega)
$$

that gives

$$
\left(\sup _{k \in D_{L}(x)}<k, \nabla(v-u)>\right)^{+} \leq(L(\nabla v)-L(\nabla u))^{+} \in \mathrm{L}^{1}(\Omega)
$$

hence $\left(\sup _{k \in D_{L}(x)}<k, \nabla(v-u)>\right)^{+} \in \mathrm{L}^{1}(\Omega)$, which combined with (3.13) gives assertion 1:

$$
\sup _{k \in D_{L}(x)}<k, \nabla(v-u)>\in \mathrm{L}^{1}(\Omega)
$$

and the proof is complete. 
We shall also need a variant of the Riesz Representation theorem:

Theorem 3.2. Let $D$ be a map from $\Omega$ to the closed convex non-empty subsets of $R \mathbb{B}$ such that $v \in\left(\mathrm{L}^{\infty}(\Omega)\right)^{n}$ implies that the map $x \mapsto m_{[D(x)-v(x)]}$ is measurable; let $T:\left(L^{1}(\Omega)\right)^{n} \rightarrow \mathbb{R}$ be a linear functional satisfying

$$
T(\xi) \leq \int_{\Omega}\left(I_{D(x)}\right)^{*}(\xi(x)) d x
$$

Then there exists $\tilde{p} \in\left(\mathrm{L}^{\infty}(\Omega)\right)^{n}, \tilde{p}(x)$ a.e. in $D(x)$, that represents $T$, i.e., such that

$$
T(\xi)=\int_{\Omega}<\tilde{p}(x), \xi(x)>d x
$$

A proof can be found on [1].

\section{The proof of Theorem 2.3}

Proof. a) Let $\eta \in C_{c}^{\infty}(\Omega)$ be arbitrary and let $\bar{v}_{t}$ and $\underline{v}_{t}$ be defined as in (3.1) and (3.2). Applying Lemma 3.1 to these variations we obtain

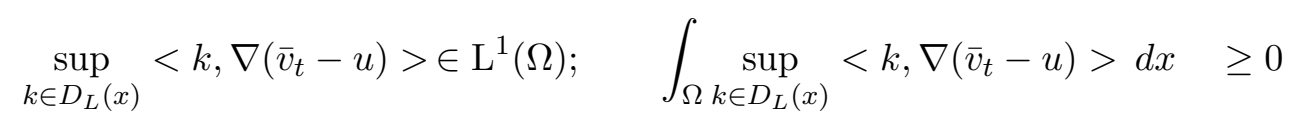

and

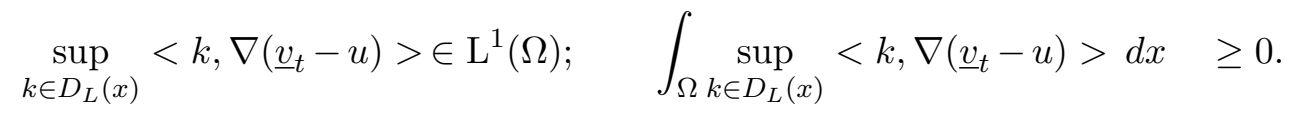

For $t>1$ consider the set $A_{t}=\left\{\eta>\frac{u+R}{t}\right\} \cap K$; since $u>-R$ on $K$, we have that $\eta(x)>0$ for $x \in A_{t}$, hence that $\nabla \eta=\nabla\left(\eta^{+}\right)$and $A_{t} \subseteq \operatorname{supt}\left(\eta^{+}\right)$. Moreover, $t \eta-R>u$ in $A_{t}$, so that, for $x$ in $A_{t}$, we have $\bar{v}_{t}=t \eta-R$ while, in $\Omega \backslash A_{t}, \bar{v}_{t}-u=0$. Then, (4.1) yields

$$
\int_{A_{t}} \sup _{k \in D_{L}(x)}<k, \nabla \eta-\frac{1}{t} \nabla u>d x=\int_{\Omega} \chi_{A_{t}} \sup _{k \in D_{L}(x)}<k, \nabla \eta-\frac{1}{t} \nabla u>d x \geq 0 .
$$

For $t \rightarrow+\infty, \nabla \eta-\frac{1}{t} \nabla u$ converges pointwise to $\nabla \eta$ and, being $t>1$, on $A_{t}$ we have

$$
\left|\sup _{k \in D_{L}(x)}<k, \nabla \eta-\frac{1}{t} \nabla u>\right|<\left|\sup _{k \in D_{L}(x)}<k, \nabla\left(\underline{v}_{t}-u\right)>\right|
$$


and the map at the right hand side is in $\mathrm{L}^{1}(\Omega)$ by (4.1).

Since $\chi_{A_{t}}$ converges to $\chi_{\operatorname{supt}\left(\eta^{+}\right)}$, by dominated convergence we conclude that

$$
\begin{gathered}
0 \leq \lim _{t \rightarrow+\infty} \int_{A_{t}} \sup _{k \in D_{L}(x)}<k, \nabla \eta-\frac{1}{t} \nabla u>d x \\
=\int_{\operatorname{supt}\left(\eta^{+}\right)} \sup _{k \in D_{L}(x)}<k, \nabla\left(\eta^{+}\right)>d x=\int_{\Omega} \sup _{k \in D_{L}(x)}<k, \nabla\left(\eta^{+}\right)>d x .
\end{gathered}
$$

Analogously, consider the set $B_{t}=\left\{\eta<\frac{u-R}{t}\right\} \cap K$; we have that $\underline{v}_{t}=t \eta+R$ in $B_{t}$. Through the same steps as before, we obtain

$$
\int_{\Omega} \sup _{k \in D_{L}(x)}<k, \nabla\left(-\eta^{-}\right)>d x \geq 0 .
$$

Add (4.3) to (4.4) to yield: for every $\eta \in C_{c}^{\infty}(\Omega)$,

$$
\int_{\Omega} \sup _{k \in D_{L}(x)}<k, \nabla \eta>d x \geq 0 .
$$

b) Choose arbitrarily $K$, a compact subset of $\Omega$, and pick $K^{\prime}, K^{\prime \prime}$ compact subsets of $\Omega$ such that $K \subseteq \operatorname{int}\left(K^{\prime}\right) \subseteq K^{\prime} \subseteq \operatorname{int}\left(K^{\prime \prime}\right)$. Let $\vartheta \in C_{c}^{\infty}(\Omega)$ be defined as in section 3 and let $v$ be defined as in (3.5). Apply Lemma 3.1 to $v$ to obtain $\sup _{k \in D_{L}(x)}<k, \nabla(v-u)>\in \mathrm{L}^{1}(\Omega)$, so that, in particular, ${\left(\sup _{k \in D_{L}(x)}<k, \nabla(v-\right.}$ $u)>) \in \mathrm{L}^{1}(K)$. Since $\nabla v=0$ on $K$, it follows that $\sup _{k \in D_{L}(x)}<k,-\nabla u>=$ $-\inf _{k \in D_{L}(x)}<k, \nabla u>\in \mathrm{L}^{1}(K)$, hence that

$$
\inf _{k \in D_{L}(x)}<k, \nabla u>\in \mathrm{L}^{1}(K)
$$

from our assumptions, $\left(\sup _{k \in D_{L}(x)}<k,-\nabla u>\right)^{+}$is bounded by an integrable function; let $x$ be such that $\left(\sup _{k \in D_{L}(x)}<k,-\nabla u>\right)^{-}>0$; then

$$
\sup _{k \in D_{L}(x)}\left\langle k,-\nabla u>\geq \inf _{k \in D_{L}(x)}<k,-\nabla u>=-\left|\inf _{k \in D_{L}(x)}<k,-\nabla u>\right|\right.
$$

so that $\left(\sup _{k \in D_{L}(x)}<k, \nabla u>\right)^{-} \leq\left|\inf _{k \in D_{L}(x)}<k,-\nabla u>\right|$, proving the claim. Since $K$ was arbitrary, we obtain

$$
\sup _{k \in D_{L}(x)}<k, \nabla u>\in \mathrm{L}_{l o c}^{1}(\Omega) .
$$

c) Let $v_{j}^{+}$and $v_{j}^{-}$be defined as in (3.6) and (3.7). We have

$$
\sup _{k \in D_{L}(x)}<k, \nabla\left(v_{j}^{+}-u\right)>\in \mathrm{L}^{1}(\Omega) \text { and } \sup _{k \in D_{L}(x)}<k, \nabla\left(v_{j}^{-}-u\right)>\in \mathrm{L}^{1}(\Omega),
$$


hence, considering $v_{j}^{+}$,

$$
\sup _{k \in D_{L}(x)}<k,-\nabla u+e_{j}>\in \mathrm{L}^{1}(K) \text { and } \sup _{k \in D_{L}(x)}<k,-\nabla u-e_{j}>\in \mathrm{L}^{1}(K),
$$

Since $\left\langle k, e_{j}\right\rangle=<k, \nabla u>+<k,-\nabla u+e_{j}>$, it follows that

$$
\sup _{k \in D_{L}(x)}<k, e_{j}>\leq \sup _{k \in D_{L}(x)}<k, \nabla u>+\sup _{k \in D_{L}(x)}<k,-\nabla u+e_{j}>
$$

and the function at the right hand side, $f^{1}$, is integrable. Considering $v_{j}^{-}$, we have

$$
\begin{gathered}
-\inf _{k \in D_{L}(x)}<k, e_{j}>=\sup _{k \in D_{L}(x)}<k,-e_{j}> \\
\leq \sup _{k \in D_{L}(x)}<k, \nabla u>+\sup _{k \in D_{L}(x)}<k,-\nabla u-e_{j}>
\end{gathered}
$$

and the function at the righ hand side, $f^{2}$, is integrable. Hence

$$
-f^{2} \leq \inf _{k \in D_{L}(x)}<k, e_{j}>\leq \sup _{k \in D_{L}(x)}<k, e_{j}>\leq f^{1}
$$

so that $\sup _{k \in D_{L}(x)}\left|k_{j}\right| \in \mathrm{L}^{1}(K)$. Since $j$ was arbitrary, $\sup _{k \in D_{L}(x)}|k| \in \mathrm{L}^{1}(K)$ and we obtain

$$
\left\|D_{L}(\cdot)\right\| \in \mathrm{L}_{l o c}^{1}(\Omega)
$$

d)Reinterpret $\sup _{k \in K}<k, \nabla \eta>$ as $\left(I_{K}\right)^{*}(\nabla \eta)$; then, the conclusion of point a) can be stated as: for every $\eta \in C_{c}^{\infty}(\Omega)$

$$
\begin{aligned}
0 & \leq \int_{\Omega} \sup _{k \in D_{L}(x)}<k, \nabla \eta>d x=\int_{\Omega} \sup _{k \in D_{L}(x)}<\frac{k}{\left\|D_{L}(x)\right\|},\left\|D_{L}(x)\right\| \nabla \eta>d x \\
& =\int_{\Omega} \sup _{h \in \frac{D_{L}(x)}{\left\|D_{L}(x)\right\|}}<h,\left\|D_{L}(x)\right\| \nabla \eta>d x=\int_{\Omega}\left(I_{\frac{D_{L}(x)}{\left\|D_{L}(x)\right\|}}\right)^{*}\left(\left\|D_{L}(x)\right\| \nabla \eta\right) d x
\end{aligned}
$$

and from (4.8) we have that $\left\|D_{L}(x)\right\| \nabla \eta \in \mathrm{L}^{1}(\Omega)$.

Consider the map

$$
\rho(\xi):=\int_{\Omega}\left(I_{\frac{D_{L}(x)}{\left\|D_{L}(x)\right\|}}\right)^{*}(\xi) d x
$$

since

$$
\left(I_{\frac{D_{L}(x)}{\left\|D_{L}(x)\right\|}}\right)^{*}(\xi)=\sup _{h \in \frac{D_{L}(x)}{\left\|D_{L}(x)\right\|}}<h, \xi>\leq \sup _{h \in \frac{D_{L}(x)}{\left\|D_{L}(x)\right\|}}|h||\xi|=|\xi(x)|,
$$


$\rho(\xi)$ is defined on $\left(\mathrm{L}^{1}(\Omega)\right)^{n}$ as a convex, positively homogeneous map.

Consider $\mathbb{L}$, the linear subspace of $\left(\mathrm{L}^{1}(\Omega)\right)^{n}$ defined as

$$
\mathbb{L}=\left\{\xi \in\left(\mathrm{L}^{1}(\Omega)\right)^{n}: \exists \eta \in C_{c}^{\infty}(\Omega): \xi=\left\|D_{L}(x)\right\| \nabla \eta\right\}
$$

and, on $\mathbb{L}$, the linear functional

$$
T(\xi) \equiv 0 .
$$

Inequality (4.9) shows that, on $\mathbb{L}, T(\xi) \leq \rho(\xi)$, so the Hahn-Banach theorem allows us to extend $T$ from $\mathbb{L}$ to $\left(\mathrm{L}^{1}(\Omega)\right)^{n}$, still satisfying $T(\xi) \leq \rho(\xi)$. (The trivial extension of $T=0$, would not, in general, satisfy this inequality since $\rho$ needs not be positive.)

e) Apply the variant of the Riesz representation theorem (theorem 3.2) to $D=$ $\frac{D_{L}(x)}{\left\|D_{L}(x)\right\|}$ and to the extension of $T$ : we obtain that $\exists \tilde{p} \in\left(\mathrm{L}^{\infty}(\Omega)\right)^{n}$ representing the extension of $T$ to $\left(\mathrm{L}^{1}(\Omega)\right)^{n}$ (and in particular representing $T$ on $\mathbb{L}$ ), such that $\tilde{p}(x) \in \frac{D_{L}(x)}{\left\|D_{L}(x)\right\|}$ a.e. on $\Omega$, i.e. such that $\tilde{p}(x)=\frac{p(x)}{\left\|D_{L}(x)\right\|}$ with $p(x) \in D_{L}(x)$. Hence, for every $\eta \in C_{c}^{\infty}(\Omega)$

$$
T(\xi) \equiv 0=\int_{\Omega}<\tilde{p}(x),\left\|D_{L}(x)\right\| \nabla \eta>d x=\int_{\Omega}<p(x), \nabla \eta>d x
$$

and the map $p(\cdot)$ is a selection from $\partial_{\xi} L(\cdot, \nabla u(\cdot))$. This proves assertion 2 and completes the proof.

\section{References}

[1] G. Bonfanti and A. Cellina, The validity of the Euler-Lagrange equation Discrete Contin. Dyn. Syst. 28 (2010), no. 2, 511â€"517

[2] M. Degiovanni and M. Marzocchi, On the Euler-Lagrange equation for functionals of the Calculus of Variations without upper growth conditions, SIAM J. Control Optim. 48 (2009), 2857-2870.

[3] R. Tyrrell Rockafellar, Convex Analysis, Princeton University Press, Princeton, NJ, 1972.

Agnese Caielli and Arrigo Cellina

Dipartimento di Matematica e Applicazioni, Università degli Studi di Milano-Bicocca, Via R. Cozzi 53,I-20125 Milano, Italy

E-mail: agnese.caielli@ars21.net and arrigo.cellina@unimib.it 